\title{
Enhanced renal ammonia excretion following volume expansion in patients with well compensated cirrhosis of the liver
}

\section{R Jalan, D Kapoor}

See end of article for authors' affiliations

Correspondence to: Dr R Jalan, Liver Failure Group, Institute of Hepatology, 69-75 Chenies Mews, London, UK; r.jalan@ucl.ac.uk

Accepted for publication 20 January 2003

\begin{abstract}
Background and aims: In patients with cirrhosis, hepatic encephalopathy is often precipitated by dehydration. This study tests the hypothesis that volume expansion in cirrhotic patients increases renal ammonia excretion.

Patients and methods: Sixteen well compensated cirrhotic patients (mean Pugh score 6.7 (SEM 0.4)) were studied after an overnight fast. One litre of $0.9 \%$ saline was administered to patients intravenously over one hour. Plasma and urinary ammonia and sodium, renal plasma flow (RPF), glomerular filtration rate (GFR), plasma renin activity (PRA), and angiotensin II (ANG II) were measured before, during, and two hours after saline infusion.

Results: Saline infusion resulted in a significant reduction in plasma ammonia (93 (SEM 7) to 56 (4) umol/l; $p<0.05)$ and RPF and GFR increased $(p<0.05)$. Urinary ammonia excretion increased $(p<0.05)$ significantly. There was a significant reduction in ANG II and PRA ( $p<0.05$ for each) and the change in ammonia excretion correlated directly with the change in urinary sodium excretion $(p<0.007)$, ANG II $(p<0.002)$, and PRA $(p<0.01)$. The mean increase in urinary ammonia excretion during the observation period was $1.08 \mathrm{mmol}$. Assuming a volume of distribution of 45 litres, the corresponding change in whole body ammonia during the same period was $1.67 \mathrm{mmol}$.

Conclusion: The results of this study suggest that volume expansion reduces plasma ammonia concentration by increasing ammonia excretion and reducing ammoniagenesis.
\end{abstract}

A mmonia is a key metabolite derived from amino acid metabolism and breakdown of nitrogenous substances in the gut, ${ }^{12}$ and present hypotheses suggest that it is of central importance in the pathogenesis of hepatic encephalopathy. Current therapeutic strategies are directed towards reducing circulating concentrations of ammonia. The two main pathways of ammonia detoxification are synthesis of urea and glutamine. In the physiological state, the liver is central in urea synthesis and this forms the basis of ammonia removal. ${ }^{3}$ The other organs capable of metabolising ammonia are the brain and skeletal muscle through synthesis of glutamine. In the presence of liver disease however, loss of functional liver mass and portocaval shunting may contribute to increased ammonia levels in plasma, thus predisposing to hepatic encephalopathy. In cirrhotic patients, skeletal muscles play an important role in the detoxification of ammonia to glutamine. ${ }^{4}$ Glutamine synthesis is however only a temporary method of ammonia detoxification. To remove ammonia from the body in a situation when urea synthesis is compromised (as happens in liver disease) requires transport of glutamine to the kidney, where it is metabolised to release ammonia, which is then excreted in urine.

Liver cirrhosis is characterised by progressive changes in systemic and renal haemodynamics. In the initial compensated state, patients have splanchnic vasodilatation and increased cardiac output but no clinical signs of fluid retention. During the decompensated phase, there is sodium retention, oedema, and ascites. These changes are accompanied by alterations in renal haemodynamics and the ability of the kidneys to excrete water and solute load, resulting in disturbances of sodium, potassium, and acid-base homeostasis. Such renal derangements are likely to have an impact on other excretory functions of the kidney such as excretion of ammonia.

In the physiological state, approximately $70 \%$ of ammonia generated by the kidney is secreted into the renal vein and
$30 \%$ excreted in urine. ${ }^{5}$ This ratio is reversed during hyperammonaemia. ${ }^{6}$ Renal handling of ammonia is also altered during metabolic acidosis and hypokalaemia. ${ }^{7-9}$ Other potent stimuli modulating ammonia synthesis, transport, and excretion by the kidneys include renal blood flow, acid-base status of the tubular cell and $\mathrm{pH}$ of the tubular lumen, luminal flow rate, and plasma angiotensin II (ANG II) levels. ${ }^{10}{ }^{11}$

We have previously shown in well compensated patients with cirrhosis that intravenous infusion of saline over one hour results in a reduction in plasma renin activity (PRA) and ANG II. ${ }^{12}$ Although patients with cirrhosis manifest the most significant disturbance in ammonia metabolism in the decompensated state, it is difficult to determine the contribution of the kidneys and in particular neurohormones such as ANG II to this disturbed metabolism because most patients are receiving treatment with diuretics and the ethical problems with saline infusion. Therefore, we chose to study patients with well compensated cirrhosis. The present study was performed to test the hypothesis that volume expansion would result in suppression of ANG II levels and result in enhanced urinary excretion of ammonia.

\section{PATIENTS AND METHODS}

\section{Subjects}

Studies were undertaken with the approval of the local research ethics committee and in accordance with the

Abbreviations: GFR, glomerular filtration rate; RPF, renal plasma flow ANG II, angiotensin II; PRA, plasma renin activity; UNaV, urinary sodium excretion; $\mathrm{UNH}_{3} \mathrm{~V}$, urinary ammonia excretion; TIPS transjugular intrahepatic portosystemic shunt stent; $\mathrm{PAH}$, para-amino hippuric acid; throughout the text, the word ammonia is used to denote ammonia $\left(\mathrm{NH}_{3}\right)$ and ammonium $\left(\mathrm{NH}_{4}^{+}\right)$unless the two entities are specified separately. 


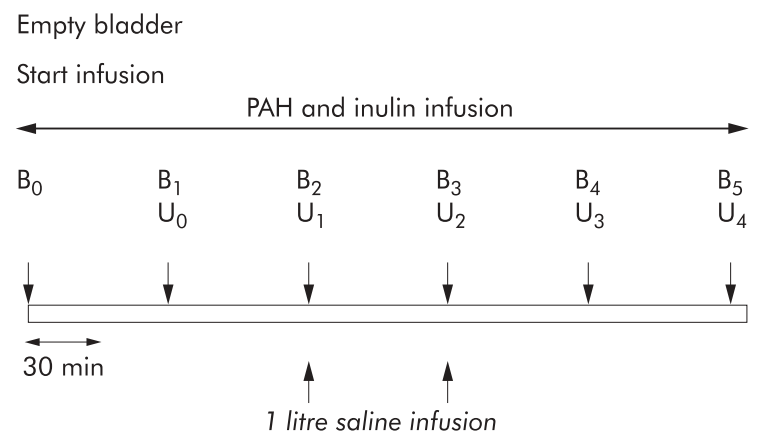

$U_{1}, U_{3}, U_{4}$ Samples for urine volume, $P A H$, inulin, ammonia; urine at $U_{0}$ and $U_{2}$ discarded

$\mathrm{B}_{1}-\mathrm{B}_{5} \quad$ Blood samples for $\mathrm{PAH}$ and inulin

$\mathrm{B}_{2}, \mathrm{~B}_{5} \quad$ Blood samples for PRA, ANG II, and ammonia

$B_{0}-B_{5} \quad 250 \mathrm{ml}$ of water orally to ensure adequate urine flow

Figure 1 Study protocol. ANG II, angiotensin II; PRA, plasma renin activity; $\mathrm{PAH}$, para-amino hippuric acid.

Declaration of Helsinki (1989) of the World Medical Association and after obtaining written informed consent from each patient.

\section{Patients}

Sixteen well compensated patients with biopsy proven cirrhosis were studied after an overnight fast and the studies were started between $0700 \mathrm{~h}$ and $0800 \mathrm{~h}$. Ten of 16 patients had a functioning transjugular intrahepatic portosystemic shunt stent (TIPS) in situ, which had been placed for variceal haemorrhage $6( \pm 1.2)$ months prior to enrolment into the current study. Shunt patency and efficacy was established by portography, with all 10 patients showing normal portal pressure gradients $(8.1(0.8) \mathrm{mm} \mathrm{Hg})$. Patients with alcoholic liver disease were required to be abstinent from alcohol for at least one month prior to enrolment in the study. Patients were excluded if they had ascites (demonstrable on ultrasonography), pitting peripheral oedema, hepatic encephalopathy, clinically evident cardiovascular disease, renal dysfunction (decreased renal size on ultrasonography, proteinuria, or creatinine concentration greater than $100 \mu \mathrm{mol} / \mathrm{l}$ ), or were receiving any diuretics or vasoactive medications. Patients were maintained on a diet with restriction of sodium intake to $150 \mathrm{mmol}$ per day for one week prior to the study. Patients were maintained in a supine posture throughout the study, adopting the erect posture only for voiding. Prior to the study, two venous cannulae were inserted, one in each arm (one for infusions and the other for sampling). Blood pressure (Dynamap, Critikon, Australia) and pulse were measured at 30 minute intervals throughout the study. Primed continuous infusion of inulin (Inutest, 25\%; Laevosan-Gesellschaft, Linz, Austria) and para-amino hippuric acid (PAH) (Merck Sharpe and Dohme, Sydney, Australia) were started as indicated and continued throughout the study. After two hours of PAH and inulin infusion, 1 litre of sodium chloride was infused over a one hour period. The timing of the infusions and various samples during the study are outlined in fig 1.

\section{Healthy volunteers}

Plasma was collected from 27 healthy volunteers with a mean age of 49 (SEM 4.1) years (16 males and 11 females) for measurement of PRA and ANG II.

\section{Measurements}

Blood was collected from a peripheral vein into pre-cooled tubes. Plasma was separated and samples stored at $-70^{\circ} \mathrm{C}$ for
Table 1 Demographics of the patients $(n=16)$

\begin{tabular}{lc}
\hline Age & $52(2.4)$ \\
Sex (M/F) & $10 / 6$ \\
Aetiology of liver disease & \\
$\quad$ Alcoholic cirrhosis & 10 \\
$\quad$ Other & 6 \\
Pugh score & $6.7(0.4)$ \\
Bilirubin (pmol/l) & $37.8(3.5)$ \\
Albumin (g/l) & $33.1(2.1)$ \\
Prothrombin time (s) & $14.9(2.1)$ \\
\hline
\end{tabular}

Data are mean (SEM).

analysis at a later date. Inulin concentration was measured using spectrophotometry, and PAH using high performance liquid chromatography. ${ }^{12} 13$

\section{Measurement of plasma renin activity}

Radioimmunoassay for measurement of PRA was based on the principle that angiotensin I is generated by the action of renin on its substrate angiotensinogen. An inhouse antibody for angiotensin I was used. The coefficient of variation for the assay was $5.2 \% .^{13}$

\section{Measurement of angiotensin II}

Samples of blood were obtained in ANG II inhibitor. ANG II values were measured by radioimmunoassay with an inhouse rabbit antibody R6B4. The coefficient of variation for the assay was $3.2 \% .^{12}$

\section{Measurement of plasma and urinary ammonia}

Blood samples were collected on ice from a cannula inserted into a peripheral vein. Plasma was obtained by centrifugation and deproteinised with trichloroacetic acid $(50 \% \mathrm{w} / \mathrm{v})$ for ammonia determination and stored at $-80^{\circ} \mathrm{C}$ for spectrophotometric determination of ammonia (CobasMiraS, HoffmanLaRoche, Switzerland). ${ }^{14}$ Urine was collected in a pre- cooled bottle which was maintained acidified with $2 \mathrm{ml}$ of $6.0 \mathrm{~N}$ $\mathrm{HCl}^{14}{ }^{14}$ The coefficient of variation for all determinations was $<4 \%$.

\section{Calculations}

Inulin clearance was used as a marker of glomerular filtration rate (GFR), and PAH clearance as a marker of renal plasma flow (RPF). Urinary sodium, urinary ammonia, and urinary volume values were used to derive the following parameters: urinary sodium excretion $(\mathrm{UNaV}, \mathrm{mmol} / \mathrm{h})$ : urinary sodiumxurinary volume;

urinary ammonia excretion $\left(\mathrm{UNH}_{3} \mathrm{~V}, \mathrm{mmol} / \mathrm{h}\right)$ : urinary ammoniaxurinary volume;

filtered ammonia load ( $\mathrm{FiNH}_{3}$ load, $\left.\mu \mathrm{mol} / \mathrm{min}\right)$ : GFR $\times$ plasma ammonia;

fractional excretion of ammonia $\left(\mathrm{FENH}_{3}, \%\right)$ : $\left(\mathrm{UNH}_{3} \mathrm{~V} / \mathrm{Fi} \mathrm{NH}\right.$ load) $\times 100$;

ammonia clearance $\left(\mathrm{NH}_{3} \mathrm{Cl}, \mathrm{ml} / \mathrm{min}\right)$ : $\mathrm{UNH}_{3} \mathrm{~V} /$ plasma ammonia.

\section{Statistical analysis}

All data are expressed as mean (SEM). Significance of differences between the patient population and healthy volunteers for PRA and ANG II were calculated using the MannWhitney test. The significance of changes within the whole study group was tested using ANOVA with repeated measures using a two tailed test to establish statistical significance. Comparison between patients with TIPS in situ and those without the stent was performed using the unpaired $t$ test or Mann-Whitney U test, wherever applicable. Changes in neurohormonal factors, plasma ammonia, urinary sodium excretion 

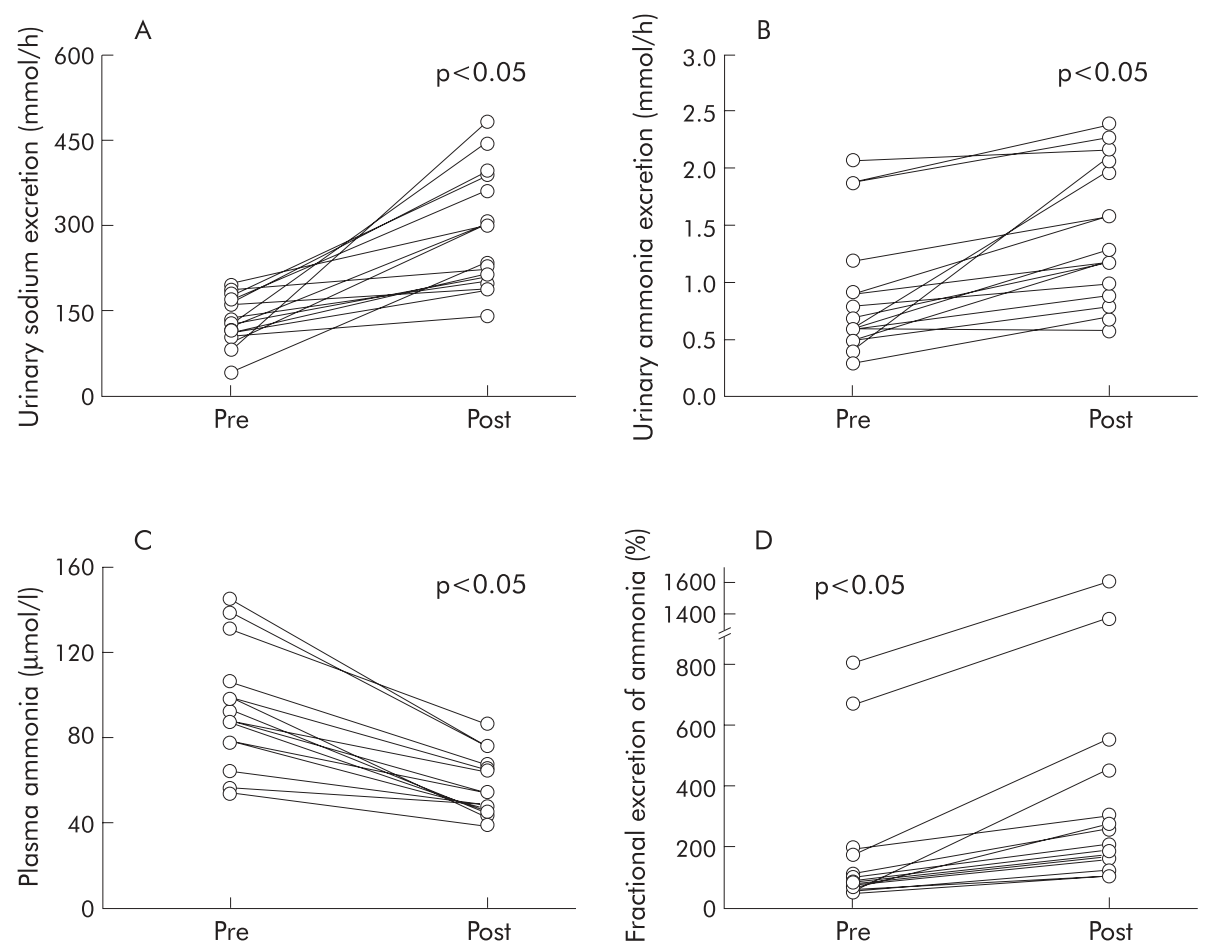

Figure 2 Individual patient data showing changes in (A) urinary sodium excretion, (B) urinary ammonia excretion, (C) plasma ammonia, and (D) fractional excretion of ammonia. Pre and post refer to time periods prior to and after infusion of 1 litre of $0.9 \%$ saline intravenously.

(UNaV), and urinary ammonia excretion $\left(\mathrm{UNH}_{3} \mathrm{~V}\right)$ were compared using linear regression.

\section{RESULTS}

The demographics of the patients are shown in table 1. At baseline, all patients had plasma ammonia levels that were greater than those in healthy volunteers $(40$ (3.9) $\mu \mathrm{mol} / \mathrm{l})$. None had signs or symptoms of clinically evident hepatic encephalopathy. There was no significant difference in plasma ammonia level between those with a TIPS and those without. Other parameters such as GFR, RPF, $\mathrm{UNH}_{3} \mathrm{~V}, \mathrm{UNaV}$, timed urine volumes, and PRA were comparable in the two subsets of patients. Only ANG II levels in patients with TIPS were significantly lower than those in patients without the stent $(\mathrm{p}<0.05)$.

Infusion of saline was not associated with any change in blood pressure, heart rate, or serum potassium values (pre 3.9 (0.3); post $3.8(0.3) \mathrm{mmol} / \mathrm{l})$. Volume expansion was associated with a significant increase in GFR (154 (17) to 181 (22) $\mathrm{ml} / \mathrm{min} ; \mathrm{p}<0.05$ ) and RPF (682 (136) to 786 (151) ml/min; $\mathrm{p}<0.05)$. Baseline values of PRA (controls $1.1(0.1)$; patients $3.3(0.8) ; \mathrm{p}<0.01$ ) and ANG II (controls 3.2 (1); patients 7.5 (1.5); $<<0.01$ ) were significantly higher in the study population compared with age matched controls. Saline infusion was associated with a significant decrease in PRA (to $1.7(0.5)$ $\mathrm{ng} / \mathrm{ml} / \mathrm{h} ; \mathrm{p}<0.05$ ) and ANG II (to $3.7(0.8) \mathrm{pg} / \mathrm{ml} ; \mathrm{p}<0.05$ ). $\mathrm{UNaV}$ increased significantly following saline infusion from 143 (19) to 293 (29) $\mathrm{mmol} / \mathrm{h}$ (fig 2A) and was similar in patients with or without TIPS.

There was a significant increase in $\mathrm{UNH}_{3} \mathrm{~V}$ from $0.9(0.1)$ to $1.4(0.2) \mathrm{mmol} / \mathrm{h}(\mathrm{p}<0.05) \quad$ (fig $2 \mathrm{~B}$ ) and a concomitant decrease in plasma ammonia levels from 93 (7) to 56 (4) $\mu \mathrm{mol} / \mathrm{l}(\mathrm{p}<0.05$ ) (fig $2 \mathrm{C}$ ). Fractional excretion of ammonia (fig 2D) and ammonia clearance ( $166(27.4)$ to $452(59.8) \mathrm{ml} / \mathrm{min}$; $\mathrm{p}<0.01)$ increased despite a decrease in $\mathrm{FiNH}_{3}(14.2$ (2.1) to $10.0(1.2) \mu \mathrm{mol} / \mathrm{min} ; \mathrm{p}=0.14)$. Changes in plasma ammonia levels as well as parameters of $\mathrm{UNH}_{3} \mathrm{~V}$ were comparable in patients with or without TIPS.

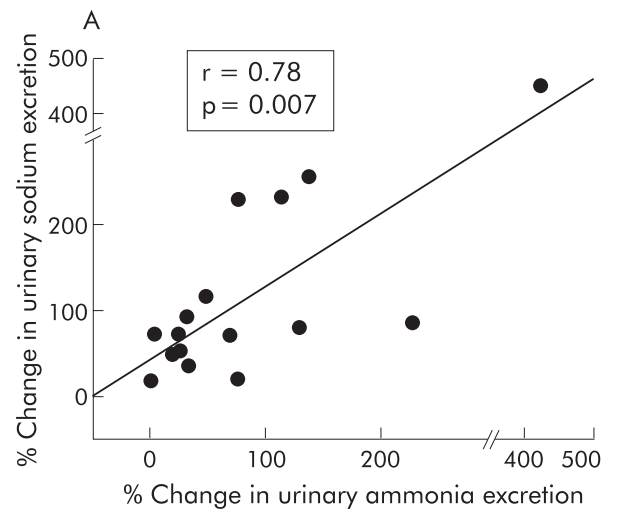

$\mathrm{B}$

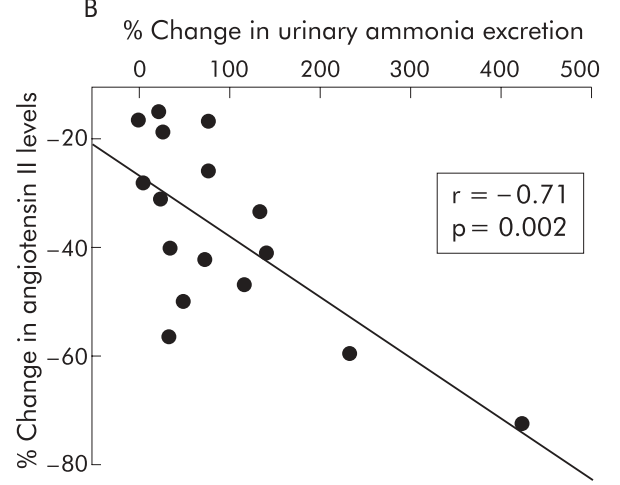

Figure 3 Scatter diagrams showing correlations between $(A)$ per cent change in urinary ammonia excretion and per cent change in urinary sodium excretion, and (B) per cent change in urinary ammonia excretion and per cent change in plasma angiotensin II concentration.

Per cent change in $\mathrm{UNH}_{3} \mathrm{~V}$ correlated positively with the per cent change in $\mathrm{UNaV}(r=0.78, \mathrm{p}<0.007)$ (fig $3 \mathrm{~A})$. The per cent 
change in $\mathrm{UNH}_{3} \mathrm{~V}$ correlated negatively with the per cent change in concentration of ANG II $(r=-0.71, \mathrm{p}<0.002)$ (fig $3 B$ ) and PRA $(p<0.02)$. The change in UNaV correlated significantly with the change in concentration of ANG II $(r=0.49, \mathrm{p}<0.04)$ but not with the change in PRA.

\section{DISCUSSION}

The results of our study show for the first time that volume expansion results in a reduction in plasma ammonia concentration and an increase in $\mathrm{UNH}_{3} \mathrm{~V}$ in patients with cirrhosis. The effect was observed in patients with a functioning TIPS as well as in those without a stent. Furthermore, volume expansion resulted in an increase in ammonia excretion, which was closely related to changes in $\mathrm{UNaV}$ and plasma ANG II concentration.

There are differing reports of the measured concentrations of circulating neurohormones in well compensated cirrhosis in the literature. While some authors have shown levels to be normal or even suppressed (especially in the supine position) ${ }_{1}^{15}{ }^{16}$ other groups including our own have shown high circulating levels of ANG II and PRA. ${ }^{12}{ }^{17-19}$ It is likely that these discrepant results reflect the differences in the technique of measurement, severity of liver disease, severity of portal hypertension, sodium intake, posture, and duration of disease. In a similar population of patients we have previously shown that infusion of 1 litre of saline resulted in suppression of elevated PRA and ANG II. ${ }^{12}$ It is against this background that we chose to study well compensated patients who were haemodynamically stable and did not have confounding factors such as a precipitating illness or concomitant diuretic usage. This enabled us to study alterations in renal ammonia handling following volume expansion and determine the concomitant changes in neurohormonal factors.

Mean plasma ammonia values in the study cohort were higher than those expected in patients with well compensated liver disease but may reflect the fact that a proportion of patients had a TIPS in situ. The high baseline values for GFR and RPF in the overall study cohort could also be due to the two subsets of patients (with and without TIPS) although values were not significantly different in the two groups. The change in GFR and estimated RPF occurred in the absence of changes in mean arterial blood pressure or heart rate and are therefore likely to have been mediated by decreases in circulating levels of ANG II and PRA.

The most striking and unequivocal change (increase) occurred in urinary excretion of ammonia with a simultaneous decrease in plasma ammonia levels. The mechanism of renal ammoniagenesis and excretion has been well established. The majority of renal ammoniagenesis occurs in the proximal convoluted tubule $e^{201}$ and is dependent on the concentration and activity of glutaminase, the concentration of glutamine reaching the proximal renal tubular cells, and luminal flow rate. ${ }^{22}$ Transport of ammonia into the tubular fluid occurs as both $\mathrm{NH}_{4}{ }^{+}$(by substitution of the hydrogen ion by the ammonium ion on the $\mathrm{Na}^{+}-\mathrm{H}^{+}$antiporter) as well as diffusion of ammonia $\left(\mathrm{NH}_{3}\right)$ across the cell membrane of the tubular cell into the acidic luminal fluid where it is trapped as $\mathrm{NH}_{4}^{+}$("diffusion trapping"). Ammonia reaching the thick ascending limb of the loop of Henle is actively absorbed into the medullary interstitium by replacement of $\mathrm{K}^{+}$with $\mathrm{NH}_{4}^{+}$on the Na-K-2Cl transporter. The concentration of ammonia in the medullary interstitium rises several fold through counter current multiplication. This ammonia is then excreted into the collecting duct and finally into urine..$^{23}$ The increased GFR and RPF that resulted from saline infusion was a possible mechanism of increasing the delivery of glutamine as well as augmenting luminal flow rate.

The mean increase in urinary ammonia observed during the study was $0.54 \mathrm{mmol} / \mathrm{h}$ or $1.08 \mathrm{mmol}$ in the two hours of the study period. If the volume of distribution of ammonia (due to its excellent permeability across lipid membranes) is considered to be equal to total body water ( $60 \%$ of total body weight or 45 litre for the study cohort), then the decrease in total body ammonia during the observation period is $1.67 \mathrm{mmol}$. Thus the decrease in total body ammonia at least during the study period exceeded that accounted for by enhanced urinary excretion of ammonia. One or more of the following mechanisms could explain this observation: (1) decreased renal and/or total body ammoniagenesis. In the former case, this would suggest that the proportion of renal ammonia production directed to the renal vein decreases while the proportion excreted in urine increases ${ }^{56} ;(2)$ unchanged renal ammoniagenesis but a decrease in only the proportion of ammonia transported into the renal vein; or (3) increased uptake of ammonia released into the circulation (through the renal vein) by the liver, skeletal muscle, and brain. In the current study, we cannot answer which of these mechanisms are operative as the fluxes of ammonia (and its substrate glutamine) across the renal and other regional circulations were not measured. In the light of recent observations by Gesek and Schoolwerth, ${ }^{25}$ Chobanian and Julin, ${ }^{26}$ and Nagami, ${ }^{27}$ we might speculate that the decrease in circulating ANG II levels induced by saline infusion could decrease renal ammonia production. These workers showed that even in the absence of changes in pH, ANG II enhanced ammoniagenesis by the proximal renal tubules in a concentration dependent manner. The observation of significant correlations between the increase in $\mathrm{UNH}_{3} \mathrm{~V}$ and decrease in ANG II is insufficient to implicate a cause-effect relationship.

The clinical relevance of the reduction in ammonia concentration by volume expansion is difficult to determine from the results of the present study and cannot be extrapolated to patients with decompensated cirrhosis. This particular study was designed on the basis of the hypothesis that has existed for over 100 years and now supported by a number of studies that "ammonia is central to the pathogenesis of hepatic encephalopathy". In addition, ammonia reduction remains the cornerstone of all of our present therapeutic armamentarium such as the use of lactulose, L-ornithine, L-aspartate, sodium benzoate, and non-absorbable antibiotics. ${ }^{28}$ We therefore believe that the reduction in ammonia by volume expansion should result in an improvement in mental state but this hypothesis will have to be tested in suitable studies.

To conclude, this is the first study to show enhanced urinary excretion of ammonia in patients with well compensated cirrhosis given an intravenous saline load. From a pathophysiological standpoint, volume loading may lead to enhanced urinary diversion of ammonia produced by the kidneys or decrease the total body/renal ammoniagenesis. Future studies should address whether this enhancement in ammonia excretion by volume expansion can be achieved in patients with decompensated cirrhosis and whether this manoeuvre results in clinical improvement of hepatic encephalopathy.

\section{ACKNOWLEDGEMENTS}

We thank Dr SWM Olde Damink, Dr NEP Deutz, and Dr CHC Dejong from the University of Maastricht for helpful discussions.

\section{Authors' affiliations}

R Jalan, D Kapoor, Liver Failure Group, Institute of Hepatology, University College London Medical School, UK,

\section{REFERENCES}

1 Jones EA, Smallwood RA, Craigie A, et al. The enterohepatic circulation of urea nitrogen. Clin Sci 1969;37:825-36.

2 Wolpert E, Phillips SF, Summerskill WH. Transport of urea and ammonia production in the human colon. Lancet 1971:2:1387-90.

3 Tannen RL. Ammonia metabolism. Am J Physiol 1978;235:F265-77.

4 Lockwood AH, McDonald JM, Reiman RE, et al. The dynamics of Lockwood AH, McDonald JM, Reiman RE, et al. The dyn
ammonia metabolism in man. Effects of liver disease and hyperammonemia. J Clin Invest 1979;63:449-60. 
5 Halperin ML, Kamel KS, Ethier JH, et al. Biochemistry and physiology of ammonia excretion. In: Seldin DW, Giebisch G, eds. The Kidney: Physiology and Pathophysiology. New York: Raven Press Ltd, 1992:2645-79.

6 Dejong CHC, Deutz NEP, Soeters PB. Renal ammonia and glutamine metabolism during liver insufficiency-induced hyperammonemia in the rat. J Clin Invest 1993;92:2834-40.

7 Gabuzda GJ, Hall PW. Relation of potassium depletion to renal ammonium metabolism and hepatic coma. Medicine 1966:45:481-90.

8 Goodman AD, Fuisz RE, Cahill GF. Renal gluconeogenesis in acidosis, alkalosis and potassium deficiency: its possible role in regulation of renal ammonia production. J Clin Invest 1966;45:612-19.

9 Schoolwerth AC. Regulation of renal ammoniagenesis in metabolic acidosis. Kidney Int 1991;40:961-73.

10 Lemieux G, Vinay P, Cartier P. Renal haemodynamics and ammoniagenesis: characteristics of the antiluminal site for glutamine extraction. J Clin Invest 1974;53:884-94.

11 Hamm LL, Simon EE. Ammonia transport in the proximal tubule. Miner Electrolyte Metab 1990;16:283-90

12 Jalan R, Hayes PC. Sodium handling in patients with well-compensated cirrhosis is dependent on the severity of liver disease and portal pressure. Gut 2000: 46:527-33.

13 Stanley AJ, Redhead DN, Bouchier IA, Hayes PC. Acute effects of transjugular intrahepatic porto-systemic stent -shunt (TIPSS) procedure on renal plasma flow and cardiopulmonary haemodynamics in cirrhosis. Am J Gastroenterol 1998;93:2463-8.

14 Dejong CHC, Deutz NEP, Soeters PB. Metabolic adaptation of the kidney to hyperammonemia during chronic liver insufficiency in the rat. Hepatology 1993;18:890-902.

15 Wilkinson SP, Smith IK, Williams R. Changes in plasma renin activity in cirrhosis: a reappraisal based on studies in 67 patients and "low-renin" cirrhosis. Hypertension 1979;2:125-9.
16 Trevisani F, Bernardi A, Gasbarrini A, et al. Bed rest induced hypernatriuresis in cirrhotic patients without ascites: does it contribute to maintain 'compensation'? J Hepatol 1992;16:190-6.

17 Bosch J, Arroyo V, Betriu A, et al. Hepatic haemodynamics and the renin- angiotensin-aldosterone system in cirrhosis. Gastroenterology 1980;78:92-9

18 Moller S, Bendtsen F, Henriksen JH. Effect of volume expansion on systemic haemodynamics and central and arterial blood volume in cirrhosis. Gastroenterology 1995:109:1917-25.

19 Helmy A, Jalan R, Newby DE, et al. Role of angiotensin II in regulation of basal and sympathetically-stimulated vascular tone in patients with early and advanced cirrhosis. Gastroenterology 2000; 1 18:565-72.

20 Knepper MA. NH4+ transport in the kidney. Kidney Int 1991;33(suppl):S95-102.

21 DuBose TD Jr, Good DW, Hamm LL, et al. Ammonium transport in the kidney: new physiological concepts and their clinical implications. J Am Soc Nephrol 1991; 1:1193-203.

22 Good DW, DuBose TD Jr. Ammonia transport by early and late proximal convoluted tubule of rat. J Clin Invest 1987;79:684-91.

23 Good DW, Knepper MA. Ammonia transport in the mammalian kidney. Am J Physiol 1985;248:F459-71.

24 Wall SM. Ammonium transport and the role of the Na, K-ATPase. Miner Electrolyte Metab 1996:22:311-17.

25 Gesek FA, Schoolwerth AC. Hormonal interactions with the proximal Na-H exchanger. Am J Physiol 1990;258:F514-21.

26 Chobanian MC, Julin CM. Angiotensin II stimulates ammoniagenesis in canine renal proximal tubule segments. Am J Physiol 1991;260:F19-26.

27 Nagami GT. Enhanced ammonia secretion by proximal tubules from mice receiving ammonium chloride: role of angiotensin II. Am J Physiol Renal Physiol 2001;282:F472-7.

28 Jalan R, Hayes PC. Hepatic encephalopathy and ascites. Lancet 1997;350:1309-15. 